Pacific Journal of Mathematic 


\title{
WEAK CHEBYSHEV SUBSPACES AND ALTERNATION
}

\author{
Frank DeUtSch, GÜNTher NÜREnberger and IVAN Singer
}

Let $T$ be a locally compact subset of $R$ and $C_{0}(T)$ the space of continuous function which vanish at infinity. An $n$ dimensional subspace $G$ of $C_{0}(T)$ may possess one of the three alternation properties:

(A-1) For each $f \in C_{0}(T)$ which has a unique best approximation $g_{0} \in G, f-g_{0}$ has $n+1$ alternating peak points;

(A-2) For each $f \in C_{0}(T)$, there exists a best approximation $g_{0} \in G$ to $f$ such that $f-g_{0}$ has $n+1$ alternating peak points;

(A-3) For each $f \in C_{0}(T)$ and each best approximation $g_{0} \in G$ to $f, f-g_{0}$ has $n+1$ alternating peak points.

In this paper, for each $i \in\{1,2,3\}$ we give an intrinsic characterization of those subspaces $G$ of $C_{0}(t)$ which have property (A-i).

1. Introduction. The classical alternation theorem states that if $G$ is an $n$ dimensional Chebyshev subspace of $C[a, b]$, then for each $f \in C[a, b]$ and its unique best approximation $g_{0} \in G$, the error $f-g_{0}$ has $n+1$ alternating peak points. It is natural to ask whether such a result remains valid if we replace $C[a, b]$ by $C(T)$, where $T$ is an arbitrary compact subset of the real line $\boldsymbol{R}$ or, more generally, by $C_{0}(T)$, where $T$ is any locally compact subset of $\boldsymbol{R}$. [Here $C_{0}(T)$ denotes the Banach space of all real-valued contionuous functions $f$ on $T$ "vanishing at infinity" (i.e., $\{t \in T|| f(t) \mid \geqq \varepsilon\}$ is compact for each $\varepsilon>0$ ), and endowed with the supremum norm: $\|f\|=\sup _{t \in T}|f(t)|$. When $T$ is actually compact, we often write $C(T)$ for $C_{0}(T)$.] And if such a result is not valid, characterize those $n$ dimensional subspaces $G$ of $C_{0}(T)$ for which the result does hold.

Properties (A-1) and (A-2) above, in the special case $T=[a, b]$, have been considered by Jones and Karlovitz [6] who proved that an $n$ dimensional subspace $G$ of $C[a, b]$ has property (A-1) if and only if $G$ has property (A-2) if and only if $G$ is "weak Chebyshev" (i.e. $G$ has property (W-4) defined below). Furthermore, Handscomb, Mayers, and Powell [5; Theorem 8] showed that an $n$ dimensional subspace $G$ of $C[a, b]$ has property (A-3) (if and) only if $G$ is a Chebyshev subspace. (The "if" part is just the classical alternation theorem.)

In this paper, for each $i \in\{1,2,3\}$, we give intrinsic characterizations of theose subspaces $G$ of $C_{0}(T)$ which have property (A-i).

It turns out that, contrary to the case when $T=[a, b]$, properties (A-1) and (A-2) are not the same in general; and property (A-3) does 
not characterize Chebyshev subspaces. In giving our characterizations of the alternation properties, the following kinds of "weak Chebyshev" subspaces play the major role. (In the definition below, the letter "W" is an abbreviation for "weak Chebyshev".)

Definition 1.1. An $n$ dimensional subspace $G$ of $C_{0}(T)$ is said have property

(W-1). If for each $1 \leqq m \leqq n$ and each set of points $-\infty=$ $t_{0}<t_{1}<\cdots<t_{m-1}<t_{m}=\infty$ with $t_{i} \in T(i=1,2, \cdots, m-1)$, there exists $0 \neq g \in G$ such that

$$
(-1)^{i} g(t) \geqq 0 \text { for all } t \in\left[t_{i}, t_{i+1}\right) \cap T \quad(i=0,1, \cdots, m-1) ;
$$

$\left(\mathrm{W}-1^{\prime}\right)$. If it satisfies the condition of property (W-1) only for $m=n$;

(W-2). If for each $1 \leqq m \leqq n$ and each set of points $-\infty=t_{0}<$ $t_{1}<\cdots<t_{m-1}<t_{m}=\infty$ with $t_{i} \in T(i=1,2, \cdots, m-1)$, there exists $0 \neq g \in G$ such that

$$
(-1)^{i} g(t) \geqq 0 \text { for } t \in\left[t_{i}, t_{i+1}\right] \cap T \quad(i=0,1, \cdots, m-1) ;
$$

$\left(\mathrm{W}-2^{\prime}\right)$. If it satisfies the condition of property (W-2) only for $m=n$;

(W-3). If for each basis $\left\{g_{1}, g_{2}, \cdots, g_{n}\right\}$ of $G$ and each set of points $t_{1}<t_{2}<\cdots t_{n}$ and $s_{1}<s_{2}<\cdots<s_{n}$ in $T$,

$$
D\left(\begin{array}{c}
g_{1} g_{2} \cdots g_{n} \\
t_{1} t_{2} \cdots t_{n}
\end{array}\right) \cdot D\left(\begin{array}{c}
g_{1} g_{2} \cdots g_{n} \\
s_{1} s_{2} \cdots
\end{array}\right) \geqq 0
$$

where

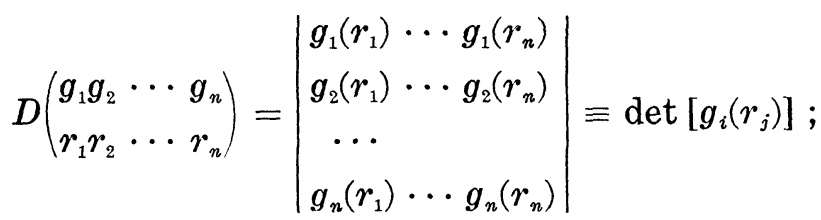

(W-4). If each $g \in G$ has at most $n-1$ sign changes, i.e., there do not exist $n+1$ points $t_{1}<t_{2}<\cdots<t_{n+1}$ in $T$ with $g\left(t_{i}\right) g\left(t_{i+1}\right)<0$ $(i=1,2, \cdots, n)$.

In $\S 2$, we study the various relationships between these weak Chebyshev properties. The main result here is Lemma 2.2. In $\S 3$, we establish that property (A-1) is equivalent to property (W-1) (Theorem 3.1). In $\S 4$, we prove that property (A-2) is equivalent to each of the (equivalent) properties (W-2), (W-2'), (W-3), and (W-4) (Theorem 4.1). In $\S 5$, we show that property (A-3) is equivalent to $G$ being Chebyshev and having one of the equivalent properties (W-2), 
(W-2'), (W-3), and (W-4) (Theorem 5.1). This allows us to give an example (Example 5.4) showing that the Handscomb, Mayers, and Powell characterization of Chebyshev subspaces is not valid in general if $T$ is not an interval. In $\S 6$, we give some examples of weak Chebyshev subspaces which are not Chebyshev. In $\S 7$, we characterize the $n$ dimensional Chebyshev subspaces of $C_{0}(T)$ for certain locally compact Hausdorff spaces $T$ (including $T$ metric, but not necessarily a subset of $\boldsymbol{R}$ ).

It is worth mentioning here the motivation for the original use of the term "weak Chebyshev". Recall the classical result that an $n$ dimensional subspace $G$ of $C[a, b]$ is Chebyshev if and only if for any basis $\left\{g_{1}, g_{2}, \cdots, g_{n}\right\}$ of $G$ and each set of points $t_{1}<t_{2}<\cdots<$ $t_{n}$ and $s_{1}<s_{2}<\cdots<s_{n}$ in $[a, b]$,

$$
D\left(\begin{array}{c}
g_{1} g_{2} \cdots g_{n} \\
t_{1} t_{2} \cdots t_{n}
\end{array}\right) \cdot D\left(\begin{array}{c}
g_{1} g_{2} \cdots g_{n} \\
s_{1} s_{2} \cdots \\
\cdots
\end{array}\right)>0 \text {. }
$$

Karlin and Studden [7] generalized this determinant criterion and defined a weak Chebyshev subspace in $C[a, b]$ as one having what we have called property (W-3). It is mainly for this historical reason that we have kept the term "weak Chebyshev subspace". However, in contrast to the case when $T=[a, b]$, not every Chebyshev subspace of $C_{0}(T)$ has property (W-3) (see Example 3.3).

We conclude the introduction by recalling some basic terminology and notation. A best approximation to $f \in C_{0}(T)$ from $G$ is any element $g_{0} \in G$ such that $\left\|f-g_{0}\right\|=\inf _{g \in G}\|f-g\|$. The set of all best approximations to $f$ from $G$ will be denoted by $P_{G}(f) . G$ is called a Chebyshev subspace if $P_{G}(f)$ is a single element for each $f \in C_{0}(T)$. An $n$ dimensional subspace $G$ of $C_{0}(T)$ is called a Haar subspace if 0 is the only element of $G$ having $n$ (or more) zeros in $T$. It is well known (at least when $T$ is compact) that $G$ is a Haar subspace if and only if it is Chebyshev. A peak point for $f \in C_{0}(T)$ is any $t \in T$ with $|f(t)|=\|f\|$. (This differs from what many authors call "peak points".) A set of points $t_{1}<t_{2}<\cdots<t_{k}$ in $T$ are called alternating peak points for $f$ if each $t_{i}$ is a peak point of $f$ and the $f\left(t_{i}\right)$ alternate in sign, i.e., $f\left(t_{i}\right)=\sigma(-1)^{i}\|f\|(i=1,2, \cdots, k)$ for some $\sigma \in\{-1,1\}$. By an interval in $R$, we shall mean any set of the form $(a, b),(a, b],[a, b)$, or $[a, b]$, where $-\infty \leqq a<b \leqq \infty$ and $a=-\infty$ or $b=\infty$ is possible on the open end. Note that every interval in $\boldsymbol{R}$ is locally compact.

Throughout this paper, unless explicitly stated otherwise, we assume that $n$ is some arbitrary but fixed positive integer and $T$ $i s$ a locally compact subset of $\boldsymbol{R}$ which contains at least $n+1$ points. 
2. Weak Chebyshev subspaces. We shall use the following topological result [1] (I. 9.7. Propositions 12 and 13, and I. 3.3, Proposition 5).

Lemma 2.1. A subset $Y$ of a locally compact Hausdorff space $X$ is locally compact $\Leftrightarrow \bar{Y} \backslash Y$ is closed. In particular, $\bar{T} \backslash T$ is closed.

It is sometimes a useful technical device to extend the functions in $C_{0}(T)$ to functions defined on the smallest closed interval IT with contains $T$ (i.e., the intersection of all closed intervals containing $T$ ). Since each function $f \in C_{0}(T)$ is uniformly continuous, it has a unique extension on to a continuous function $\widetilde{f}$ on $\bar{T}$. Obviously, we must have $\widetilde{f}=0$ on $\bar{T} \backslash T$. Since $R \backslash \bar{T}$ is open, it has a unique representation as a countable union of disjoint open intervals. Hence also $I T \backslash \bar{T}=$ $\mathrm{U}_{j} I_{j}$, where $\left(I_{j}\right)$ is a countable collection of disjoint open intervals. We now define $\tilde{f}$ on $I T \backslash \bar{T}$ by extending $\tilde{f}$ linearly across each interval $I_{j}$. It is easy to verify that the resulting function $\tilde{f}$ is in $C_{0}(I T)$.

Summarizing, each $f \in C_{0}(T)$ can be extended to a unique function $\widetilde{f} \in C_{0}(I T)$ defined by $\widetilde{f}=0$ on $\widetilde{T} \backslash T$ and $\widetilde{f}$ is linear on each of the disjoint open subintervals whose union is $I T \backslash \bar{T}$. In the sequel, the notation $\widetilde{f}$ will be reserved for this unique extension of $f$ to all of $I T$, and we let $\widetilde{G}=\{\widetilde{g} \mid g \in G\}$ denote the extension of the corresponding subspace $G$.

Lemma 2.2. Let $G$ be an $n$ dimensional subspace of $C_{0}(T)$. Consider the following statements:

(1) G has property (W-1);

(1') $G$ has property (W-1');

(2) $G$ has property (W-2);

(2') G has property (W-2');

(3) $G$ has property (W-3);

(4) $G$ has property (W-4).

Then $\left(1^{\prime}\right) \Longleftarrow(1) \Leftarrow(2) \Leftrightarrow\left(2^{\prime}\right) \Leftrightarrow(3) \Leftrightarrow(4)$. Moreover, $(1) \nrightarrow(2)$ and $\left(1^{\prime}\right) \nRightarrow(1)$ in general. In the case $n=1$, all the properties are equivalent to the existence of a nonzero function $g \in G$ with $g(t) \geqq 0$ for all $t \in T$.

Proof. The last statement is obvious as are the implications $(2) \Rightarrow\left(2^{\prime}\right)$ and $(2) \Rightarrow(1) \Rightarrow\left(1^{\prime}\right)$.

$\left(2^{\prime}\right) \Rightarrow(3)$. Let $s_{1}<s_{2}<\cdots<s_{n}$ in $T$ be such that $D\left(\begin{array}{c}g_{1} g_{2} \cdots g_{n} \\ s_{1} s_{2} \cdots s_{n}\end{array}\right) \neq$

0 . For each integer $k \in\{1,2, \cdots, n\}$ define $u_{k} \in G$ by

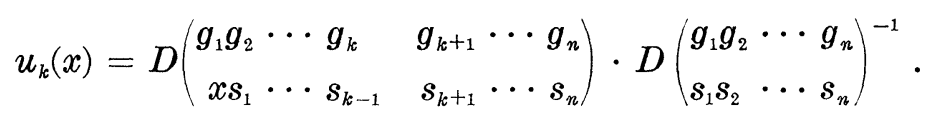


Note that

$$
u_{k}\left(s_{i}\right)=(-1)^{k-1} \delta_{k i} \quad(i, k=1,2, \cdots, n) .
$$

Set $x_{0}=-\infty, x_{i}=s_{i}(i=1, \cdots, k-1), x_{i}=s_{i+1}(i=k, \cdots, n-1)$, and $x_{n}=\infty$. By property $\left(\mathrm{W}-2^{\prime}\right)$, there exists $0 \neq v_{k} \in G$ such that

$$
(-1)^{i} v_{k}(x) \geqq 0 \text { for all } x \in\left[x_{i}, x_{i+1}\right] \cap T
$$

$(i=0,1, \cdots, n-1)$. In particular, $v_{k}\left(s_{i}\right)=0$ for all $i \neq k$ and $(-1)^{k-1} v_{k}\left(s_{k}\right) \geqq 0$. Since $\left\{u_{1}, u_{2}, \cdots, u_{n}\right\}$ is a basis for $G$, it follows using eq. (2) that $v_{k}=\lambda_{k} u_{k}$ for some $\lambda_{k}>0$.

Now let $t_{1}<t_{2}<\cdots<t_{n}$ in $T$ with $D\left(\begin{array}{c}g_{1} g_{2} \cdots g_{n} \\ t_{1} t_{2} \cdots t_{n}\end{array}\right) \neq 0$. Suppose there is a $k$ such that $s_{k} \notin\left\{t_{1}, t_{2}, \cdots, t_{n}\right\}$. Since $u_{k} \neq 0$, it follows that there is an $m$ such that $u_{k}\left(t_{m}\right) \neq 0$. From eq. (2), $t_{m} \notin\left\{s_{1}, \cdots\right.$, $\left.s_{k-1}, s_{k+1}, \cdots, s_{n}\right\}$. Set $\left\{r_{1}, r_{2}, \cdots, r_{n}\right\}=\left\{s_{1}, \cdots, s_{k-1}, s_{k+1}, \cdots, s_{n}\right\} \cup\left\{t_{m}\right\}$ with $r_{i}<r_{i+1}$ for all $i$. Then $t_{m} \in\left(x_{i}, x_{i+1}\right)$ for some $i \in\{0,1, \cdots, n-1\}$ implies

$$
\begin{aligned}
& 0 \leqq(-1)^{i} v_{k}\left(t_{m}\right)=(-1)^{i} \lambda_{k} u_{k}\left(t_{m}\right) \\
& =\lambda_{k} D\left(\begin{array}{lll}
g_{1} g_{2} \cdots & g_{n} \\
r_{1} r_{2} & \cdots & r_{n}
\end{array}\right) \cdot D\left(\begin{array}{lll}
g_{1} g_{2} & \cdots & g_{n} \\
s_{1} s_{2} & \cdots & s_{n}
\end{array}\right)^{-1}
\end{aligned}
$$

so

$$
D\left(\begin{array}{l}
g_{1} g_{2} \cdots g_{n} \\
r_{1} r_{2} \cdots \\
r_{n}
\end{array}\right) \cdot D\left(\begin{array}{l}
g_{1} g_{2} \cdots g_{n} \\
s_{1} s_{2} \cdots s_{n}
\end{array}\right)>0
$$

By a repeated application of this argument, we obtain that

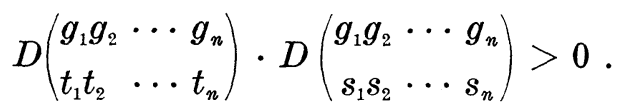

Thus $G$ has property (W-3).

At this point it is convenient to isolate some useful facts which will simplify the proof of Lemma 2.2 and are of independent interest.

Claim 1. $G$ has property (W-3) (resp. $((\mathrm{W}-4))$ in $C_{0}(T) \Leftrightarrow \widetilde{G}$ has property $(\mathrm{W}-3)$ (resp. (W-4)) in $C_{0}(I T)$.

Proof of Claim 1. If $\widetilde{G}$ has property (W-3)(resp. (W-4)) in $C_{0}(I T)$, then the restriction $G=\left.\widetilde{G}\right|_{T}$ obviously has property (W-3) (resp. (W-4)) in $C_{0}(T)$.

Next suppose $G$ has property (W-3) in $C_{0}(T)$. If $\widetilde{G}$ fails to have property (W-3) in $C_{0}(I T)$, there exist points $\widetilde{s}_{1}<\widetilde{s}_{2}<\cdots<\widetilde{s}_{n}$ and $\widetilde{t}_{1}<\widetilde{t}_{2}<\cdots<\widetilde{t}_{n}$ in $I T$ such that 


$$
D\left(\begin{array}{lll}
\widetilde{g}_{1} \cdots & \widetilde{g}_{n} \\
\widetilde{\boldsymbol{g}}_{1} \cdots & \cdots & \widetilde{\boldsymbol{s}}_{n}
\end{array}\right)<0<D\left(\begin{array}{lll}
\widetilde{\boldsymbol{g}}_{1} & \cdots & \widetilde{g}_{n} \\
\widetilde{t}_{1} \cdots & \widetilde{t}_{n}
\end{array}\right) .
$$

Let $k$ be the smallest index such that $\widetilde{t}_{k} \notin T$, and set $t_{i}=\widetilde{t}_{i} \in T$ $(i=1,2, \cdots, k-1)$. Define

$$
\widetilde{g}(t)=D\left(\begin{array}{c}
\widetilde{g}_{1} \cdots \widetilde{g}_{k-1} \widetilde{g}_{k} \widetilde{g}_{k+1} \cdots \widetilde{g}_{n} \\
t_{1} \cdots t_{k-1} t \widetilde{t}_{k+1} \cdots \tilde{t}_{n}
\end{array}\right) \quad(t \in I T) .
$$

Then $\widetilde{g} \in \widetilde{G}$ and $\widetilde{g}\left(\widetilde{t}_{k}\right)>0$. Now $\widetilde{t}_{k} \in I_{m}=(a, b)$ for some open interval $I_{m}$ (where $I T \backslash \bar{T}=\bigcup_{1}^{\infty} I_{j}$ ), and $\widetilde{g}$ is linear on $I_{m}$ implies $\widetilde{g}(a)>0$ or $\widetilde{g}(b)>0$. We may assume $\widetilde{g}(a)>0$. But the endpoints of $I_{m}$ lie in the boundary of $I T \backslash \bar{T}$, and hence in $\bar{T}$. Since $\widetilde{g}=0$ on $\bar{T} \backslash T, a \in T$. Set $t_{k}=a$. Then

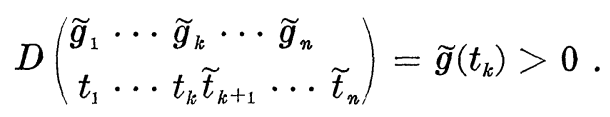

Continuing in this way with $\tilde{t}_{k+1}, \cdots, \tilde{t}_{n}$, we obtain points $t_{1}<t_{2}<$ $\cdots<t_{n}$ in $T$ such that

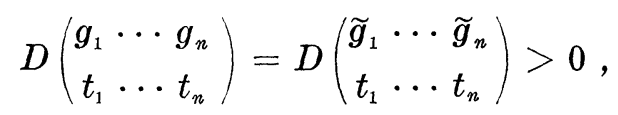

where $g_{i}=\left.\widetilde{g}_{i}\right|_{T} \in G$. Similarly, we obtain points $s_{1}<s_{2}<\cdots<s_{n}$ in $T$ such that

$$
D\left(\begin{array}{c}
g_{1} \cdots g_{n} \\
s_{1} \cdots s_{n}
\end{array}\right)<0
$$

But this contradicts $G$ having property (W-3). Thus $\widetilde{G}$ must have property (W-3) in $C_{0}(I T)$.

Now let $G$ have property (W-4) in $C_{0}(T)$. If $\widetilde{G}$ fails to have property (W-4) in $C_{0}(I T)$, there exist $\widetilde{g} \in \widetilde{G}$ and points $\widetilde{t}_{1}<\widetilde{t}_{2}<\cdots<$ $\widetilde{t}_{n}$ in $I T$ such that $\widetilde{g}\left(\tilde{t}_{i}\right) \widetilde{g}\left(\widetilde{t}_{i+1}\right)<0(i=1,2, \cdots, n)$. If all $\tilde{t}_{i}$ are in $T$, then the function $g=\left.\widetilde{g}\right|_{T} \in G$ satisfies

$$
g\left(\tilde{t}_{i}\right) g\left(\tilde{t}_{i+1}\right)<0 \quad(i=1,2, \cdots, n)
$$

which contradicts $G$ having property (W-4) in $C_{0}(T)$. Thus let $k$ be the smallest index such that $\widetilde{t}_{k} \notin T$ and set $t_{i}=\widetilde{t}_{i} \in T$ for $i=1,2, \cdots$, $k-1$. Since $\widetilde{g}=0$ on $\bar{T} \backslash T, \tilde{t}_{k} \in I T \backslash \bar{T}$ so $\widetilde{t}_{k} \in I_{m}$ for some open interval $I_{m}$. We may assume $\widetilde{g}\left(\tilde{t}_{k}\right)<0$. By the same argument as in the above proof of the implication " $G$ has $(\mathrm{W}-3) \Rightarrow \widetilde{G}$ has $(\mathrm{W}-3)$ ", we obtain a point $t_{k} \in T$ such that

$$
t_{1}<t_{2}<\cdots<t_{k}<\tilde{t}_{k+1}<\cdots<\tilde{t}_{n+1}
$$


and $\widetilde{g}\left(t_{k}\right)<0$. Continuing in this way with $\widetilde{t}_{k+1}, \cdots, \widetilde{t}_{n+1}$, we obtain points $t_{1}<t_{2}<\cdots<t_{n+1}$ in $T$ such that

$$
g\left(t_{i}\right) g\left(t_{i+1}\right)=\widetilde{g}\left(t_{i}\right) \widetilde{g}\left(t_{i+1}\right)<0 \quad(i=1,2, \cdots, n),
$$

where $g=\left.\widetilde{g}\right|_{T} \in G$. But this contradicts $G$ having property (W-4) in $C_{0}(T)$.

ClaIm 2. Let $I$ be an interval in $R, H$ an $n$ dimensional subspace of $C_{0}(I)$, and $\left(I_{j}\right)$ an increasing sequence of compact intervals such that $I=\bigcup_{1}^{\infty} I_{j}$ and $\left.H\right|_{I_{1}}$ is $n$ dimensional. Then, for any given $i \epsilon$ $\left\{1,1^{\prime}, 2,2^{\prime}, 3,4\right\}, H$ has property (W-i) in $\left.C_{0}(I) \Leftrightarrow H\right|_{I_{j}}$ has property $(\mathrm{W}-\mathrm{i})$ in $C_{0}\left(I_{j}\right)$ for each $j$.

Proof of Claim 2. Clearly, if $H$ has property (W-i) in $C_{0}(I)$, then the restriction $\left.H\right|_{I_{j}}$ has property $(\mathrm{W}-\mathrm{i})$ in $C_{0}\left(I_{j}\right)$ for each $j$.

Conversely, suppose first that $\left.H\right|_{I_{j}}$ has property (W-2') in $C_{0}\left(I_{j}\right)$ for each $j$. Let $-\infty=t_{0}<t_{1}<\cdots<t_{n-1}<t_{n}=\infty$, where $t_{i} \in I(i=$ $1,2, \cdots, n-1)$. Choose $N$ sufficiently large that $t_{i} \in I_{N}(i=1,2, \cdots$, $n-1)$. For each $k \geqq N$ there exists $\left.h_{k} \in H\right|_{I_{k}} \mid\{0\}$ such that

$$
(-1)^{i} h_{k}(t) \geqq 0 \text { for all } t \in\left[t_{i}, t_{i+1}\right] \cap I_{k}
$$

$(i=0,1, \cdots, n-1)$. Choose $g_{k} \in H$ such that $h_{k}=\left.g_{k}\right|_{I_{k}}$ and let $g$ be a cluster point of the sequence $\left(g_{k} /\left\|g_{k}\right\|\right)$. Then $g \in H \backslash\{0\}$ and

$$
(-1)^{i} g(t) \geqq 0 \text { for all } t \in\left[t_{i}, t_{i+1}\right] \cap I
$$

$(i=0,1, \cdots, n-1)$. Thus $H$ has property $\left(\mathrm{W}-2^{\prime}\right)$ in $C_{0}(I)$.

The proof of the implication " $\Longleftarrow$ " in the case when $i=1,1^{\prime}$, or 2 is similar to the case $i=2^{\prime}$ proved above.

Next, assume that $\left.H\right|_{I_{j}}$ has property (W-4) in $C_{0}\left(I_{j}\right)$ for each $j$. If $H$ fails to have property (W-4) in $C_{0}(I)$, there exist points $t_{1}<$ $t_{2}<\cdots<t_{n}$ in $I$ and $h \in H$ such that $h\left(t_{i}\right) h\left(t_{i+1}\right)<0(i=1,2, \cdots, n)$. Choose $N$ sufficiently large that $t_{i} \in I_{N}$ for all $i$. Then $h_{N}=\left.h\right|_{I_{N}} \in$ $\left.H\right|_{I_{N}}$ satisfies

$$
h_{N}\left(t_{i}\right) h_{N}\left(t_{i+1}\right)<0 \quad(i=1,2, \cdots, n)
$$

which contradicts $\left.H\right|_{I_{N}}$ having property (W-4) in $C_{0}\left(I_{N}\right)$. Thus $H$ has property (W-4) in $C_{0}(I)$.

The proof of the implication "£" in the case when $i=3$ is similar to the above proof when $i=4$.

Claim 3. Let $I$ be an interval in $\boldsymbol{R}$ and $H$ an $n$ dimensional subspace of $C_{0}(I)$. Then $H$ has one of the properties (W-2'), (W-3), or $(\mathrm{W}-4) \Leftrightarrow H$ has them all. 
Proof of Claim 3. There exists an increasing sequence of compact intervals $\left(I_{j}\right)$ such that $I=\bigcup_{1}^{\infty} I_{j}$ and $\left.H\right|_{I_{1}}$ is $n$ dimensional. By Jones-Karlovitz [6], $\left.H\right|_{I_{j}}$ has one of the properties (W-2'), (W-3), or (W-4) in $\left.C_{0}\left(I_{j}\right) \Leftrightarrow H\right|_{I_{j}}$ has them all. The result now follows from Claim 2.

Claim 4. Let $I$ be an interval in $R$ and $H$ an $n$ dimensional subspace of $C_{0}(I)$. Then $H$ has property $\left(\mathrm{W}-2^{\prime}\right) \Leftrightarrow H$ has property (W-2).

Proof of Claim 4. The implication " $\Longleftarrow "$ is obvious. Thus assume $H$ has property $\left(\mathrm{W}-2^{\prime}\right)$. Let $1 \leqq m \leqq n$ and $-\infty=t_{0}<t_{1}<\cdots<$ $t_{m-1}<t_{m}=\infty$ with $t_{i} \in I(i=1,2, \cdots, m-1)$. We may assme $m<n$. If $t_{m-1}<\sup I$, choose points $t_{m}^{(k)}<t_{m+1}^{(k)}<\cdots<t_{n-1}^{(k)}<t_{n}^{(k)}=\infty$, with $t_{m-1}<t_{m}^{(k)}, t_{i}^{(k)} \in I(i=m, m+1, \cdots, n-1)$, and $t_{m}^{(k)} \rightarrow \sup I$ as $k \rightarrow \infty$. Define $t_{i}^{(k)}=t_{i}$ if $0 \leqq i \leqq m-1$. If $t_{m-1}=\sup I$, choose points $t_{m-1}^{(k)}<$ $t_{m}^{(k)}<\cdots<t_{n-1}^{(k)}<t_{n}^{(k)}=\infty$, with $t_{m-2}<t_{m-1}^{(k)}, t_{i}^{(k)} \in I(i=m-1, m, \cdots$, $n-1)$, and $t_{m-1}^{(k)} \rightarrow \sup I$ as $k \rightarrow \infty$. Define $t_{i}^{(k)}=t_{i}$ if $0 \leqq i \leqq m-2$. In either case, there exists $h_{k} \in H \backslash\{0\}$ such that

$$
(-1)^{i} h_{k}(t) \geqq 0 \text { for all } t \in\left[t_{i}^{(k)}, t_{i+1}^{(k)}\right] \cap I
$$

$(i=0,1, \cdots, n-1)$, and all $k$. Let $h$ be any cluster point of the sequence $\left(h_{k} /\left\|h_{k}\right\|\right)$. Then $h \in H \backslash\{0\}$ and

$$
(-1)^{i} h(t) \geqq 0 \text { for all } t \in\left[t_{i}, t_{i+1}\right] \cap I
$$

$(i=0,1, \cdots, m-1)$. Hence $H$ has property $(W-2)$.

We can now easily complete the proof of Lemma 2.2 .

$(3) \Rightarrow(4)$. If $G$ has property $(\mathrm{W}-3)$ in $C_{0}(T)$, then Claim 1 implies $\widetilde{G}$ has property (W-3) in $C_{0}(I T)$. By Claim $3, \widetilde{G}$ has property (W-4) in $C_{0}(I T)$. By Claim $1, G$ has property (W-4) in $C_{0}(T)$.

$(4) \Rightarrow(2)$. If $G$ has property (W-4) in $C_{0}(T)$, then $\breve{G}$ has property $(\mathrm{W}-4)$ in $C_{0}(I T)$ by Claim 1 . By Claim $3, \widetilde{G}$ has property $\left(\mathrm{W}-2^{\prime}\right)$ in $C_{0}(I T)$. By Claim 4, $\widetilde{G}$ has property $(\mathrm{W}-2)$ in $C_{0}(I T)$. Clearly, $G=$ $\left.\widetilde{G}\right|_{T}$ has property $(\mathrm{W}-2)$ in $C_{0}(T)$.

We show that $(1) \nsim(2)$ and $\left(1^{\prime}\right) \nsim(1)$ in examples below. This completes the proof.

The proof of the implication $\left(2^{\prime}\right) \Rightarrow(3)$ is an obvious modification of the proof given in [6] for the special case $T=[a, b]$. The implications $\left(2^{\prime}\right) \Rightarrow(3) \Leftrightarrow(4)$ have been verified independently by Zielke [10] using a different argument, and in the more general setting with $G$ any $n$ dimensional subspace of $\boldsymbol{R}^{T}$ : the set of all real-valued functions on $T$, where $T$ is any subset of $\boldsymbol{R}$. 
The following two examples show that the the implications $(1) \Rightarrow$ (2) and $\left(1^{\prime}\right) \Rightarrow(1)$ in Lemma 2.2 are false in general.

2.3. Example of a subspace having property $\left(\mathrm{W}-1^{\prime}\right)$ but not (W-1). Consider the set $T=\{1,2,3,4\}$ and $G=\operatorname{span}\left\{g_{1}, g_{2}, g_{3}\right\} \subset C(T)$, where $g_{1}=\delta_{1}-\delta_{4}, g_{2}=\delta_{2}-\delta_{4}, g_{3}=\delta_{3}-\delta_{4}$, and $\delta_{i}(j)=1$ if $i=j, 0$ if $i \neq j$. It is easy to see that there is no $g \neq 0$ in $G$ such that $g \geqq 0$. Thus $G$ fails (W-1). To see that $G$ has property (W-1'), we show that for each pair of points $t_{1}<t_{2}$ in $T$, there is a nonzero $g \in G$ such that $(-1)^{i} g(t) \geqq 0$ for all $t \in\left[t_{i}, t_{i+1}\right) \cap T(i=0,1,2)$ (where $t_{0}=$ $-\infty$ and $\left.t_{3}=\infty\right)$. We list all the possible choices of $t_{1}<t_{2}$ and the corresponding $g$ below. If $\left\{t_{1}, t_{2}\right\}=\{1,2\},\{1,3\}$, or $\{1,4\}$, take $g=-g_{1}$. If $\left\{t_{1}, t_{2}\right\}=\{2,3\}$ or $\{2,4\}$, take $g=g_{1}-g_{2}$. If $\left\{t_{1}, t_{2}\right\}=\{3,4\}$, take $g=-g_{3}$.

2.4. Example of a subspace having property (W-1) but not (W-2). Let $T$ be the set of natural numbers and let $G=\operatorname{span}\left\{g_{1}, g_{2}\right\} \subset$ $C_{0}(T)\left(=c_{0}\right)$, where $g_{1}=\delta_{1}, g_{2}=\delta_{2}-\delta_{3}$, and $\delta_{i}(j)=1$ if $i=j, 0$ otherwise. It is easy to check that $G$ has property (W-1). However, the function $g=g_{1}-g_{2}$ has two sign changes so $G$ fails $(\mathrm{W}-4)$. By Lemma 2.2, $G$ fails (W-2).

Under certain conditions on $T$ (e.g., if $T$ is an interval or if $T$ is unbounded), the properties (W-1) and (W-1') are equivalent. This is the content of the following result.

Proposition 2.5. Suppose that either $T$ is unbounded or inf $T$ or $\sup T$ is an accumulation point of $T$ (in $R$ ). Then an $n$ dimensional subspace $G$ of $C_{0}(T)$ has property $(\mathrm{W}-1) \Leftrightarrow$ it has property $\left(\mathrm{W}-1^{\prime}\right)$.

Proof. By Lemma 2.2 it suffices to verify that $\left(\mathrm{W}-1^{\prime}\right) \Rightarrow(\mathrm{W}-1)$. Let $G$ have property (W-1'). If $\sup T$ is an accumulation point of $T$ or if $\sup T=\infty$, then the same proof as given in Claim 4 of Lemma 2.2 shows that $G$ has property (W-1). If inf $T$ is an accumulation point of $T$ or if $\inf T=-\infty$, a similar proof works.

We next give a condition which insures that property (W-1) is equivalent to (W-2).

DEFINITION 2.6. A function $\delta: T \rightarrow R$ is called a delta function if $\delta$ is the characteristic function of a point in $T$. That is, for some $t_{0} \in T, \delta=\chi_{t_{0}}$, where $\chi_{t_{0}}(t)=0$ if $t \neq t_{0}$ and $\chi_{t_{0}}\left(t_{0}\right)=1$.

Since a delta function $\chi_{t_{0}}$ is continuous iff $t_{0}$ is an isolated point of $T, C_{0}(T)$ contains delta functions iff $T$ contains isolated points. 
Observe also that a subspace $G$ of $C_{0}(T)$ cannot contain a delta function if some $f \in C_{0}(T) \backslash G$ has a unique best approximation $g_{0} \in G$. (For otherwise, some scalar multiple of the delta function, added to $g_{0}$, would another best approximation to $f$.) In particular, a Chebyshev subspace of $C_{0}(T)$ cannot contain delta functions.

Proposition 2.7. Let $G$ be an $n$ dimensional subspace of $C_{0}(T)$ which does not contain any delta function. Then $G$ has property $(\mathrm{W}-1) \Leftrightarrow G$ has property (W-2).

Proof. It suffices by Lemma 2.2 to show that if $G$ has property (W-1), it has property (W-2). Fix any integer $m, 1 \leqq m \leqq n$. We must show that for each set of points $-\infty=t_{0}<t_{\mathrm{i}}<\cdots<t_{m}=\infty$ with $t_{i} \in T(i=1, \cdots, m-1)$, there exists $0 \neq g \in G$ such that

(a) $(-1)^{i} g(t) \geqq 0$ for all $t \in\left[t_{2}, t_{i+1}\right) \cap T(i=0,1, \cdots, m-1)$ and

(b) $g\left(t_{i}\right)=0(i=1,2, \cdots, m-1)$.

If $m=1$, condition (b) is vacuously satisfied and (a) follows by property (W-1). Thus we may assume $m>1$. What we will show is that for each integer $k$, with $1 \leqq k \leqq m-1$, and each set of points $-\infty=t_{0}<t_{1}<\cdots<t_{m}=\infty$ with $t_{\imath} \in T(i=1, \cdots, m-1)$, there is $0 \neq g_{k} \in G$ such that $(-1)^{i} g_{k}(t) \geqq 0$ for all $t \in\left[t_{i}, t_{i+1}\right) \cap T(i=0,1, \cdots$, $m-1)$ and $g_{k}\left(t_{i}\right)=0(i=1,2, \cdots, k)$. Then the function $g=g_{m-1}$ will satisfy (a) and (b). We proceed by induction on $k$.

Assume first that $k=1$. By property (W-1), there exists $0 \neq$ $g \in G$ such that (a) holds. If $g\left(t_{1}\right)=0$, set $g_{1}=g$ and we are done. If $g\left(t_{1}\right) \neq 0$, then $g\left(t_{1}\right)<0$.

Case 1. $t_{1}=\sup T$.

Choose $g_{0} \in G \backslash\{0\}$ such that $g_{0}(t) \geqq 0$ for all $t \in T$.

Case 2. $t_{1}<\sup T$.

Let $\tau=\inf \left\{t \in T \mid t>t_{1}\right\}$. Then $t_{1} \leqq \tau \leqq t_{2}$.

We consider three subcases.

Case 2.1. $\tau=t_{1}$.

Choose a sequence $\left(\tau_{j}\right)$ in $T, t_{1}<\tau_{j}<t_{2}$, such that $\tau_{j} \rightarrow t_{1}=\tau$. Set $t_{i}^{(j)}=t_{i}$ if $i \neq 1$ and $t_{1}^{(j)}=\tau_{j}$. Choose $g^{(j)} \in G,\left\|g^{(j)}\right\|=1$, such that $(-1)^{i} g^{(j)}(t) \geqq 0$ for all $t \in\left[t_{i}^{(j)}, t_{i+1}^{(j)}\right) \cap T(i=0,1, \cdots, m-1)$. Let $g_{0}$ be a cluster point of the sequence $\left(g^{(j)}\right)$.

Case 2.2. $\tau=t_{2}$.

Set $\tilde{t}_{0}=-\infty$ and $\tilde{t}_{i}=t_{i+2}(i=1,2, \cdots, m-2)$. Choose $0 \neq g_{0} \in$ $G$ such that $(-1)^{i} g_{0}(t) \geqq 0$ for all $t \in\left[\tilde{t}_{i}, \tilde{t}_{i+1}\right] \cap T(i=0,1, \cdots, m-3)$. 
Case 2.3. $t_{1}<\tau<t_{2}$.

If $\tau \in T$, let $\tilde{t}_{i}=t_{i}$ if $i \neq 1$ and $\tilde{t}_{1}=\tau$. Choose $0 \neq g_{0} \in G$ such that $(-1)^{i} g_{0}(t) \geqq 0$ for all $t \in\left[\tilde{t}_{i}, \tilde{t}_{i+1}\right) \cap T(i=0,1, \cdots, m-1)$.

If $\tau \notin T$, choose a sequence $\left(\tau_{j}\right)$ in $T$ with $\tau<\tau_{j}<t_{2}$ and $\tau_{j} \rightarrow \tau$. Set $t_{i}^{(j)}=t_{i}$ if $i \neq 1$ and $t_{1}^{(j)}=\tau_{j}$. Choose $g^{(j)} \in G,\left\|g^{(j)}\right\|=1$, such that $(-1)^{i} g^{(j)}(t) \geqq 0$ for all $t \in\left[t_{i}^{(j)}, t_{i+1}^{(j)}\right) \cap T(i=0,1, \cdots, m-1)$. Let $g_{0}$ be a cluster point of the sequence $\left(g^{(j)}\right)$.

In every case, we have obtained a function $g_{0} \in G \backslash\{0\}$ satisfying $g_{0}(t) g(t) \geqq 0$ for all $t \in T \backslash\left\{t_{1}\right\}$ and $g_{0}\left(t_{1}\right) \geqq 0$. If $g_{0}\left(t_{1}\right)=0$, set $g_{1}=g_{0}$. If $g_{0}\left(t_{1}\right) \neq 0$, then $g_{0}\left(t_{1}\right)>0$ and set $g_{1}=g-\left(g\left(t_{1}\right) / g_{0}\left(t_{1}\right)\right) g_{0}$. Since $\alpha=$ $g\left(t_{1}\right) / g_{0}\left(t_{1}\right)<0$, it follows that $g_{1}$ satisfies $(-1)^{i} g_{1}(t) \geqq 0$ for all $t \epsilon$ $\left[t_{i}, t_{i+1}\right) \cap T(i=0,1, \cdots, m-1)$, and $g_{1}\left(t_{1}\right)=0$. If $g_{1}=0$, then $g=$ $\alpha g_{0}$. Since $G$ contains no delta functions, there exists a point $\widetilde{t} \epsilon$ $T \backslash\left\{t_{1}\right\}$ such that $g_{0}(\tilde{t}) \neq 0$. Hence

$$
0 \leqq g(\widetilde{t}) g_{0}(\tilde{t})=\alpha\left[g_{0}(\tilde{t})\right]^{2}<0
$$

which is absurd. Thus $g_{1} \neq 0$ and the proof of the first step $k=1$ is complete.

The proof of the induction step is analogous.

REMARK 2.8. Example 2.3 above is of a subspace $G$ which does not contain any delta function, has property (W-1'), but not (W-1), and hence not (W-2), (W-2'), (W-3), or (W-4).

CoROllary 2.9. Let $I$ be any interval in $\boldsymbol{R}$ and let $G$ be $n$ dimensional subspace of $C_{0}(I)$. Then $G$ has one of the following properties $\Leftrightarrow$ it has then all: (W-1), (W-1'), (W-2), (W-2'), (W-3), (W-4).

Proof. Since $I$ contains no isolated point, $C_{0}(I)$ contains no delta function.

If $I$ were bounded, then both sup $I$ and inf $I$ would be accumulation points of $I$. The result now follows by Lemma 2.2, Proposition 2.5, and Proposition 2.7.

We note that when $I$ is an interval, the equivalent properties (W-1), (W-1'), (W-2), and (W-2') simplify somewhat. For example, if $I$ is a bounded interval and $a=\inf (I), b=\sup (I)$, then an $n$ dimensional subspace $G$ of $C_{0}(I)$ has property (W-2') iff for each set of points

$$
a=t_{0}<t_{1}<\cdots<t_{n-1}<t_{n}=b,
$$

there exists $g \in G \backslash\{0\}$ such that

$$
(-1)^{i} g(t) \geqq 0 \text { for all } t \in\left[t_{i}, t_{i+1}\right]
$$


$(i=0,1, \cdots, n-1)$

\section{A characterization of property (A-1).}

THEOREM 3.1. Let $G$ be an $n$ dimensional subspace of $C_{0}(T)$. The following statements are equivalent:

(1) G has property (W-1);

(2) For each $f \in C_{0}(T)$ which has a unique best approximation $g_{0} \in G, f-g_{0}$ has at least $n+1$ alternating peak points;

(3) Each $f \in C_{0}(T)$ which has 0 as its unique best approximation in $G$ must have at least $n+1$ alternating peak points.

REMARK. In particular, if $G$ is "very non-Chebyshev" (i.e., each $f \in C_{0}(T) \backslash G$ has more than one best approximation in $G$ ), then $G$ has property $(\mathrm{W}-1)$.

Proof. $\quad(1) \Rightarrow(2)$. Suppose $G$ has property (W-1). Fix any $f \in$ $C_{0}(T)$ with $P_{G}(f)=\left\{g_{0}\right\}$ a singleton. If $f=g_{0}$, any $n+1$ points in $T$ work. Thus we assume $f \neq g_{0}$. Choose a compact set $K$ in $T$ so that

$$
\left|\left(f-g_{0}\right)(t)\right|<\frac{1}{2}\left\|f-g_{0}\right\| \text { for all } t \in T \backslash K .
$$

We define a set of points in $T$ inductively as follows. Let

$$
t_{1}=\min \left\{t \in K||\left(f-g_{0}\right)(t) \mid=\left\|f-g_{0}\right\|\right\} .
$$

We may assume $\left(f-g_{0}\right)\left(t_{1}\right)=\left\|f-g_{0}\right\|$. Having chosen $t_{i}$, we set

$$
t_{i+1}=\min \left\{t \in K \cap\left[t_{i}, \infty\right) \mid\left(f-g_{0}\right)(t)=-\left(f-g_{0}\right)\left(t_{i}\right)\right\} .
$$

Now either this procedure yields $n+1$ points $t_{i}$ (which clearly satisfy $\left.(-1)^{i+1}\left(f-g_{0}\right)\left(t_{i}\right)=\left\|f-g_{0}\right\|\right)$ and we are done, or this process ends with $m$ points $t_{i}, 1 \leqq m \leqq n$ (i.e., the set $K \cap\left[t_{m}, \infty\right) \cap\left\{t \in T \mid\left(f-g_{0}\right)(t)=\right.$ $\left.-\left(f-g_{0}\right)\left(t_{m}\right)\right\}$ is empty $)$. Thus we assume the latter case.

Set $z_{0}=-\infty$ and $z_{m}=\infty$. If $m>1$ we define additional $z_{i}$ as follows: for each $i=1,2, \cdots, m-1$, set

$$
z_{i}=\max \left\{t \in K \cap\left[t_{i}, t_{i+1}\right] \mid(-1)^{i+1}\left(f-g_{0}\right)(t) \geqq 0\right\} .
$$

It follows that $t_{i} \leqq z_{i}<t_{i+1}$ and so

$$
-\infty=z_{0}<z_{1}<\cdots<z_{m-1}<z_{m}=\infty .
$$

For $i=0,1, \cdots, m-1$, let 


$$
M_{i}=\max \left\{(-1)^{i+1}\left(f-g_{0}\right)(t) \mid t \in\left[z_{i}, z_{i+1}\right] \cap K\right\}
$$

and $M=\max \left\{M_{i} \mid i=0,1, \cdots, m-1\right\}(\geqq 0)$. By compactness of $K$ and the choice of the $t_{i}$ and $z_{i}$, it follows that $M<\left\|f-g_{0}\right\|$.

Since $G$ has property (W-1), there is $0 \neq g \in G$ such that $(-1)^{i+1} g(t) \geqq 0$ for all $t \in\left[z_{i}, z_{i+1}\right) \cap T(i=0,1, \cdots, m-1)$. By scaling $g$ we may assume

$$
0<\|g\| \leqq \min \left\{\frac{1}{2}\left\|f-g_{0}\right\|,\left\|f-g_{0}\right\|-M\right\} .
$$

If $t \in T \backslash K$, then

$$
\begin{aligned}
\left|\left(f-g_{0}-g\right)(t)\right| & \leqq\left|\left(f-g_{0}\right)(t)\right|+|g(t)|<\frac{1}{2}\left\|f-g_{0}\right\|+\|g\| \\
& \leqq\left\|f-g_{0}\right\| .
\end{aligned}
$$

If $t \in\left[z_{i}, z_{i+1}\right) \cap K(i=0,1, \cdots, m-1)$, then

$$
\begin{aligned}
(-1)^{i+1}\left(f-g_{0}-g\right)(t) & =(-1)^{i+1}\left(f-g_{0}\right)(t)-(-1)^{i+1} g(t) \\
& \leqq M+\|g\| \leqq\left\|f-g_{0}\right\|
\end{aligned}
$$

and

$$
(-1)^{i+1}\left(f-g_{0}-g\right)(t) \geqq(-1)^{i+1}\left(f-g_{0}\right)(t) \geqq-\left\|f-g_{0}\right\| .
$$

Thus $\left|\left(f-g_{0}-g\right)(t)\right| \leqq\left\|f-g_{0}\right\|$ implies that $\left\|f-g_{0}-g\right\| \leqq\left\|f-g_{0}\right\|$ and hence $g_{0}+g \in P_{G}(f)=\left\{g_{0}\right\}$, a contradiction.

$(2) \Rightarrow(3)$ is obvious.

$(3) \Rightarrow(1)$. Assume (3) holds and let $1 \leqq m \leqq n$ and $-\infty=t_{0}<$ $t_{1}<\cdots<t_{m-1}<t_{m}=\infty$, where $t_{i} \in T(i=1,2, \cdots, m-1)$. We show there exists $g \in G \backslash\{0\}$ such that $(-1)^{i} g(t) \geqq 0$ for all $t \in\left[t_{i}, t_{i+1}\right) \cap T$ $(i=0,1, \cdots, m-1)$. Let $T_{0}=\bar{T} \backslash T$ and for each positive integer $N$, let

$$
T_{N}=\left\{t \in T \mid \operatorname{dist}\left(t, T_{0}\right) \geqq \frac{1}{N}\right\}
$$

if $T_{0} \neq \varnothing$ and $T_{N}=T$ if $T_{0}=\varnothing$. It is easy to verify that $T_{N}$ is a closed subset of $\boldsymbol{R}$, hence of $T, T_{N} \subset T_{N+1}$, and $T=\bigcup_{1}^{\infty} T_{N}$.

Thus the sets

$$
\begin{aligned}
& K_{N, 0}=\left[t_{1}-N, t_{1}-\frac{1}{N}\right] \cap T_{N}, \\
& K_{N, i}=\left[t_{i}, t_{i+1}-\frac{1}{N}\right] \cap T_{N} \quad(i=1,2, \cdots, m-2),
\end{aligned}
$$

and 


$$
K_{N, m}=\left[t_{m-1}, t_{m-1}+N\right] \cap T_{N}
$$

are disjoint compact subsets of $T$. $\left(K_{N, 0}\right.$ may be empty for all $N$ in which case we simply ignore it in the subsequent argument.) By Urysohn's lemma (or our linearization procedure) there exists functions $f_{N, i} \in C_{0}(T)(i=0,1, \cdots, m-1)$ such that $0 \leqq f_{N, i} \leqq 1, f_{N, i}=1$ on $K_{N, i}$, $f_{N, i}=0$ off $\left(t_{i}-1 / 2 N, t_{i+1}-1 / 2 N\right)(i=1,2, \cdots, m-2), f_{N, 0}=0$ off $\left(t_{1}-N-1 / N, t_{1}-1 / 2 N\right)$, and $f_{N, m-1}=0$ off $\left(t_{m-1}-1 / N, t_{m-1}+N+1 / N\right)$. Set $f_{N}=\sum_{i=0}^{m-1}(-1)^{i} f_{N, i}$. Then $f_{N} \in C_{0}(T)$ and $f_{N}$ has only $m-1 \leqq$ $n-1$ changes of sign so $f_{N}$ has at most $n$ alternating peak points. Thus 0 cannot be the unique best approximation to $f_{N}$. Choose any $g_{N} \in P_{G}\left(f_{N}\right) \backslash\{0\}$. Then $g_{N}$ must have the same sign as $f_{N}$ on the sets $K_{N, i}$. That is, $(-1)^{i} g_{N}(t) \geqq 0$ for all $t \in K_{N, i}(i=0,1, \cdots, m-1)$. Let $h_{N}=g_{N} /\left\|g_{N}\right\|$. Let $g$ be a cluster point of $\left(h_{N}\right)$. Then $g \in G,\|g\|=$ 1. Since $K_{N, 0} \nearrow\left(-\infty, t_{1}\right) \cap T$ as $N \rightarrow \infty, K_{N, i} \nearrow\left[t_{i}, t_{i+1}\right) \cap T(i=1,2$, $\cdots, m-2)$, and $K_{N, m} \nearrow\left[t_{m-1}, \infty\right) \cap T$, it follows that $(-1)^{i} g(t) \geqq 0$ for all $t \in\left[t_{i}, t_{i+1}\right) \cap T(i=0,1, \cdots, m-1)$.

Corollary 3.2. Let $G$ be an $n$ dimensional subspace of $C_{0}(T)$ which does not contain any delta function (e.g., if $T$ is an interval or if $G$ is Chebyshev). Then:

(a) The following statements are equivalent:

(1) $G$ has any one of the equivalent properties (W-1), (W-2), (W-2'), (W-3), or (W-4);

(2) For each $f \in C_{0}(T)$ which has a unique best approximation $g_{0} \in G, f-g_{0}$ has at least $n+1$ alternating peak points;

(3) East $f \in C_{0}(T)$ having 0 as its unique best approximation in $G$ has at least $n+1$ alternating peak points.

(b) If $T$ satisfies the hypothesis of Proposition 2.5 (e.g., if $T$ is an interval), and if $G$ is a Chebyshev subspace, then the above statements are equivalent to

(4) $G$ has property (W-1').

(c) If $T$ is an interval and $G$ is a Chebyshev subspace, then $G$ has all of the weak Chebyshev properties (W-1), (W-1'), (W-2), (W-2'), (W-3), and (W-4).

Proof. The proof of (a) follows from Theorem 3.1, Lemma 2.2, and Proposition 2.7.

The proof of (b) follows from part (a) and Proposition 2.5.

To prove (c), let $T$ be an interval and $G$ a Chebyshev subspace. Then for each $f \in C_{0}(T), f-P_{G}(f)$ has $n+1$ alternating peak points. (This follows, essentially, by a result of Bram [2]. It can also be 
deduced just as Remez did in the classical case $T=[a, b]$; see e.g., [8].) The result now follows from (b).

There seems to be a commonly held belief that statement (2) of Corollary 3.2 is always valid for Chebyshev subspaces $G$ of $C(T)$, if $T$ is a compact subset of $\boldsymbol{R}$. The following example shows this to be false.

3.3. Example of a one dimensional Chebyshev subspace of $C(T)$ which fails to have any weak Chebyshev property. Let $T=$ $\{1,2\}$. Consider the one dimensional subspace $G$ spanned by the function $g_{1}$ defined by $g_{1}(t)=(-1)^{t}(t \in T)$. Then $G$ is Chebyshev since $g_{1}$ has no zeros. Since $g_{1}$ changes sign, it follows by Lemma 2.2 (when $n=1$ ) that $G$ fails to have any of the weak Chebyshev properties. Hence by Corollary 3.2(a), some $f \in C_{0}(T)$ has the property that $f-P_{G}(f)$ has less than two alternating peak points. (More explicity, define $f \in C_{0}(T)$ by $f(1)=0$ and $f(2)=2$. Then $P_{G}(f)=g_{1}$ and $f-g_{1}$ is of one sign.)

\section{A characterization of property (A-2).}

THEOREM 4.1. Let $G$ be an $n$ dimensional subspace of $C_{0}(T)$. The following statements are equivalent.

(1) $G$ has any one of the equivalent properties (W-2), (W-2'), (W-3), or (W-4);

(2) For each $f \in C_{0}(T)$, there exists a $g_{0} \in P_{G}(f)$ such that $f-g_{0}$ has at least $n+1$ alternating peak points.

Proof. $(1) \Rightarrow(2)$. Suppose $G=\operatorname{span}\left\{g_{1}, g_{2}, \cdots, g_{n}\right\}$ has property (W-4) and $f \in C_{0}(T) \backslash G$. Assume first that $T$ is a compact interval $[a, b]$. Then the result follows from Jones-Karlovitz [6]. Next let $T$ be an arbitrary interval. Then there is an increasing sequence of compact intervals $T_{k}$ such that $\bigcup_{1}^{\infty} T_{k}=T$. For $k$ sufficiently large, $\left.G\right|_{T_{k}}$ will be $n$ dimensional and we assume this to be the case. Set $G_{k}=\left.G\right|_{T_{k}}$. Then each $G_{k}$ has property (W-4) (in $\left.C_{0}(T)\right|_{T_{k}} \subset C\left(T_{k}\right)$ ) since $G$ does. By the first part, there exist $h_{k} \in P_{G_{k}}\left(\left.f\right|_{T_{k}}\right)$ and $n+1$ points $t_{k_{1}}<t_{k_{2}}<\cdots<t_{k, n+1}$ in $T_{k}$ such that

$$
\sigma_{k}(-1)^{i}\left(f-h_{k}\right)\left(t_{k i}\right)=\left\|f-h_{k}\right\|_{k} \quad(i=1,2, \cdots, n+1)
$$

for some $\sigma_{k} \in\{-1,1\}$, where $\|h\|_{k}=\|h\|_{T_{k}}=\sup _{t \in T_{k}}|h(t)| \quad$ Since $h_{k} \in P_{G_{k}}\left(\left.f\right|_{T_{k}}\right)$, we have

$$
\left\|h_{k}\right\|_{k} \leqq 2\|f\|_{k} \leqq 2\|f\| \text { for all } k \text {. }
$$

By passing to a subsequence, we may assume all the $\sigma_{k}$ to be the 
same, say $\sigma_{k}=1$. Write $h_{k}=\left.\sum_{i=1}^{n} \alpha_{k i} g_{i}\right|_{T_{k}}$.

Claim. $\sup _{k}\left|\alpha_{k i}\right|<\infty(i=1,2, \cdots, n)$.

Indeed, we may assume that $\operatorname{dim} G_{1}=n . \quad$ Since $T_{1} \subset T_{k}$ and $h_{k} \in P_{G_{k}}\left(\left.f\right|_{T_{k}}\right)(k=1,2, \cdots)$, we have

$$
\left\|f-h_{k}\right\|_{1} \leqq\left\|f-h_{k}\right\|_{k} \leqq\|f\|_{k} \leqq\|f\| \quad(k=1,2, \cdots),
$$

so

$$
\left\|h_{k}\right\|_{1} \leqq\|f\|+\|f\|_{1} \leqq 2\|f\| \quad(k=1,2, \cdots) .
$$

Now $G_{1}$ is isomorphic to $l_{\infty}^{n}$ (the space of all $n$-tuples of real numbers endowed with the maximum norm) by the mapping

$$
\left.\sum_{i=1}^{n} \beta_{i} g_{i}\right|_{T_{1}} \longrightarrow\left(\beta_{1}, \beta_{2}, \cdots, \beta_{n}\right) \text {. }
$$

Hence there exists a constant $M_{n}$ (depending only on $n$ ) such that

$$
\max _{1 \leqq i \leqq n}\left|\beta_{i}\right| \leqq M_{n}\left\|\left.\sum_{i=1}^{n} \beta_{i} g_{i}\right|_{T_{1}}\right\|
$$

for all real numbers $\beta_{1}, \beta_{2}, \cdots, \beta_{n}$. Since $\left.h_{k}\right|_{T_{1}}=\left.\sum_{i=1}^{n} \alpha_{k i} g_{i}\right|_{T_{1}}$ and $\left\|h_{k}\right\|_{1} \leqq 2\|f\|$, we obtain

$$
\max _{1 \leqq i \leqq n}\left|\beta_{k i}\right| \leqq M_{n}\left\|h_{k}\right\|_{1} \leqq 2 M_{n}\|f\| \quad(k=1,2, \cdots)
$$

from which the claim follows.

Using the claim, we can pass to a subsequence and obtain $\alpha_{k i} \rightarrow$ $\alpha_{i}(i=1,2, \cdots, n)$ for some $\alpha_{i} \in R$. Let $g_{0}=\sum_{1}^{n} \alpha_{i} g_{i} \in G$ and $\bar{h}_{k}=$ $\sum_{i=1}^{n} \alpha_{k i} g_{i} \in G$. Then $\left.\bar{h}_{k}\right|_{T_{k}}=h_{k}$ and $\bar{h}_{k} \rightarrow g_{0}$. Since $\left.\bar{h}_{k}\right|_{T_{k}}=h_{k} \in P_{G_{k}}\left(\left.f\right|_{T_{k}}\right)$, we have,

$$
\left\|f-\bar{h}_{k}\right\|_{k} \leqq\left\|f-g_{0}\right\|_{k} \leqq\left\|f-g_{0}\right\| \quad(k=1,2, \cdots) .
$$

On the other hand, we can choose $t_{0} \in T$ such that $\left|\left(f-g_{0}\right)\left(t_{0}\right)\right|=$ $\left\|f-g_{0}\right\|$. Since $\bigcup_{1}^{\infty} T_{k}=T$ and the $T_{k}$ are increasing, we have $t_{0} \in T_{k}$ for all $k$ sufficiently large. Also $\bar{h}_{k} \rightarrow g_{0}$ implies that for each $\varepsilon>0$, there exists $k_{\varepsilon}$ such that $\left|\left(f-\bar{h}_{k}\right)\left(t_{0}\right)\right| \geqq\left|\left(f-g_{0}\right)\left(t_{0}\right)\right|-\varepsilon$ for all $k \geqq k_{\varepsilon}$. Thus for all $k$ sufficiently large,

$$
\left\|f-\bar{h}_{k}\right\|_{k} \geqq\left|\left(f-\bar{h}_{k}\right)\left(t_{0}\right)\right| \geqq\left|\left(f-g_{0}\right)\left(t_{0}\right)\right|-\varepsilon=\left\|f-g_{0}\right\|-\varepsilon .
$$

Hence $\left\|f-\bar{h}_{k}\right\|_{k} \rightarrow\left\|f-g_{0}\right\|$. Thus for $i=1,2, \cdots, n+1$,

$$
\begin{aligned}
\left|\left(f-g_{0}\right)\left(t_{k i}\right)\right| & =\left|\left(f-\bar{h}_{k}\right)\left(t_{k i}\right)-\left(g_{0}-\bar{h}_{k}\right)\left(t_{k i}\right)\right| \\
& \geqq\left\|f-\bar{h}_{k}\right\|_{k}-\left\|g_{0}-\bar{h}_{k}\right\|_{k} \longrightarrow\left\|f-g_{0}\right\|,
\end{aligned}
$$

and hence 


$$
t_{k i} \in K \equiv\left\{t \in T||\left(f-g_{0}\right)(t) \mid \geqq \frac{1}{2}\left\|f-g_{0}\right\|\right\}
$$

for $k$ sufficiently large. Since $K$ is compact, by passing to a subsequence, we may assume that $t_{k i} \rightarrow t_{i} \in T(i=1,2, \cdots, n+1)$.

By passing to the limit in eq. (1),

$$
(-1)^{i}\left(f-g_{0}\right)\left(t_{i}\right)=\left\|f-g_{0}\right\| \quad(i=1,2, \cdots, n+1),
$$

and $t_{1}<t_{2}<\cdots<t_{n+1}$. If $g_{0} \notin P_{G}(f)$, then for any $g \in P_{G}(f)$ we obtain $\left|(f-g)\left(t_{i}\right)\right|<\left\|f-g_{0}\right\|$ and

$$
\begin{aligned}
(-1)^{i}\left(g-g_{0}\right)\left(t_{i}\right) & =(-1)^{i}\left(f-g_{0}\right)\left(t_{i}\right)-(-1)^{i}(f-g)\left(t_{i}\right) \\
& =\left\|f-g_{0}\right\|-(-1)^{i}(f-g)\left(t_{i}\right)>0
\end{aligned}
$$

for $i=1,2, \cdots, n+1$. Thus $g-g_{0}$ has $n$ sign changes, contradicting property (W-4). This shows that $g_{0} \in P_{G}(f)$ and proves the result when $T$ is any interval.

Finally, let $T$ be any locally compact subset of $\boldsymbol{R}$. Let $I T, \widetilde{f} \in$ $C_{0}(I T)$, and $\widetilde{G}$ be as described prior to Lemma 2.2. Since $G$ has property (W-4) and since each $\widetilde{g} \in \widetilde{G}$ is linear, hence monotonic, on each interval in $I T \backslash \bar{T}, \widetilde{G}$ also has property (W-4). Fix any $f \in$ $C_{0}(T) \backslash G$. Then $\widetilde{f} \in C_{0}(I T) \backslash \widetilde{G}$ and by the result proved for intervals there exist $\widetilde{g}_{0} \in P_{\widetilde{G}}(\widetilde{f})$ and points $\widetilde{t}_{1}<\widetilde{t}_{2}<\cdots<\widetilde{t}_{n+1}$ in $I T$ such that

$$
\sigma(-1)^{i}\left(\tilde{f}-\widetilde{g}_{0}\right)\left(\widetilde{t}_{i}\right)=\left\|\tilde{f}-\widetilde{g}_{0}\right\|_{I^{T}} \neq 0 \quad(i=1,2, \cdots, n+1)
$$

for some $\sigma \in\{-1,1\}$.

Claim. We may assume $\widetilde{t}_{i} \in T(i=1,2, \cdots, n+1)$.

If $\tilde{t}_{i} \in T$, set $t_{i}=\widetilde{t}_{i}$. If some $\widetilde{t}_{i} \notin T$, then (since $\widetilde{f}-\widetilde{g}_{0}=0$ on $\bar{T} \backslash T$ ) $\widetilde{t}_{i}$ is in one of the disjoint open intervals $I_{m}$ whose union is $I T \backslash \bar{T}$. But $\widetilde{f}-\widetilde{g}_{0}$ is linear on each such subinterval and $\left|\left(\tilde{f}-\widetilde{g}_{0}\right)\left(\widetilde{t}_{i}\right)\right|=\left\|\widetilde{f}-\widetilde{g}_{0}\right\|_{I T}$ imply that $\tilde{f}-\widetilde{g}_{0}$ is constant on $I_{m}$. We then replace $\widetilde{t}_{i}$ with either one of the endpoints $t_{i}$ of $I_{m}$. Clearly, the resulting $t_{i} \in T, t_{1}<t_{2}<$ $\cdots<t_{n+1}$, and

$$
\sigma(-1)^{i}\left(\tilde{f}-\widetilde{g}_{0}\right)\left(t_{i}\right)=\left\|\tilde{f}-\widetilde{g}_{0}\right\|_{I T} \quad(i=1,2, \cdots, n+1)
$$

which proves the claim.

Since $\left\|\widetilde{f}-\widetilde{g}_{0}\right\|_{I T} \leqq\|\widetilde{f}-\widetilde{g}\|_{I T}$ for all $\widetilde{g} \in \widetilde{G}$ and since $\|\widetilde{h}\|_{I T}=\|h\|$ for every $h \in C_{0}(T)$ (because $\tilde{h}$ is linear on each of the subintervals whose union is $I T \backslash \bar{T})$, it follows that $\left\|f-g_{0}\right\| \leqq\|f-g\|$ for all $g \in G$. That is, $g_{0} \in P_{G}(f)$, and using the claim,

$$
\sigma(-1)^{i}\left(f-g_{0}\right)\left(t_{i}\right)=\left\|f-g_{0}\right\| \quad(i=1,2, \cdots, n+1) .
$$

$(2) \Rightarrow(1)$. Suppose (2) holds. We will show that $G$ has property $\left(\mathrm{W}-2^{\prime}\right)$. We first prove the 
Claim. For each set of points $-\infty=t_{0}<t_{1}<\cdots<t_{n}=\infty$, with $t_{i} \in T(i=1,2, \cdots, n-1)$, there exists $g \in G \backslash\{0\}$ such that

\section{$(-1)^{2} g(t) \geqq 0$ for all $t \in\left[t_{i}, t_{i+1}\right) \cap T$}

$(i=1,2, \cdots, n-1)$, and $g(t) \neq 0$ for some $t \in T \backslash\left\{t_{1}, t_{2}, \cdots, t_{n-1}\right\}$.

We construct the sets $K_{N, i}(i=0,1, \cdots, n)$ and functions $f_{N} \in C_{0}(T)$ exactly as in the proof of the implication (3) $\Rightarrow(1)$ of Theorem 3.1. Then $f_{N}$ has at most $n$ alternating peak points. Choose $g_{N} \in P_{G}\left(f_{N}\right)$ such that $f_{N}-g_{N}$ has at least $n+1$ alternating peak points. Then $g_{N} \neq 0$ and $g_{N} f_{N} \geqq 0$ on each set $K_{N, 2}(i=0,1, \cdots, n)$. Let $h_{N}=$ $\left(g_{N} /\left\|g_{N}\right\|\right)$ and let $g$ be a cluster point of the sequence $\left(h_{N}\right)$. Then $g \in G,\|g\|=1$, and $(-1)^{i} g(t) \geqq 0$ for all $t \in\left[t_{i}, t_{\imath+1}\right) \cap T(i=0,1, \cdots$, $n-1)$. If $g(t)=0$ for all $t \in T \backslash\left\{t_{1}, t_{2}, \cdots, t_{n-1}\right\}$, then for such $t,\left|h_{N}(t)\right|<1 / 8$ eventually (i.e., for $N$ large enough) and hence $\left|g_{N}(t)\right|<$ $1 / 4$ eventually (since $\left\|g_{N}\right\| \leqq 2\left\|f_{N}\right\| \leqq 2$ ). But if $t \in T \backslash\left\{t_{1}, t_{2}, \cdots, t_{n-1}\right\}$, then $t \in K_{N, i}$ eventually for some $i$ implies that, eventually,

$$
\left|f_{N}(t)-g_{N}(t)\right| \geqq\left|f_{N}(t)\right|-\left|g_{N}(t)\right| \geqq 1-\frac{1}{4}=\frac{3}{4} .
$$

Thus for some integer $N_{0}$, we have $\left\|f_{N_{0}}-g_{N_{0}}\right\| \geqq 3 / 4$. It follows using the definition of $f_{N_{0}}$ and the above properties of $g_{N_{0}}$ that $f_{N_{0}}$ $g_{N_{0}}$ cannot have more than $n$ alternating peak points, a contradiction to the choice of $g_{N}$. This proves the claim.

We next show that for each set of points $-\infty=t_{0}<t_{1}<\cdots<$ $t_{n-1}<t_{n}=\infty$, with $t_{i} \in T(i=1,2, \cdots, n-1)$, and each integer $k$, with $1 \leqq k \leqq n-1$, there exists $g_{k} \in G \backslash\{0\}$ such that

(a) $(-1)^{i} g_{k}(t) \geqq 0$ for all $t \in\left[t_{i}, t_{i+1}\right) \cap T(i=0,1, \cdots, n-1)$, and (b) $g_{k}\left(t_{i}\right)=0(i=1,2, \cdots, k)$.

Once we have this, it is clear that the function $g=g_{n-1}$ satisfies

$$
(-1)^{i} g(t) \geqq 0 \text { for all } t \in\left[t_{i}, t_{i+1}\right] \cap T
$$

$(i=0,1, \cdots, n-1)$ and this shows that $G$ has property $\left(\mathrm{W}-2^{\prime}\right)$. We proceed by induction on $k$. The induction step is similar to the case $k=1$ so we only prove the latter. By the claim, there exist $g \in$ $G \backslash\{0\}$ which satisfies (a) and $g(s) \neq 0$ for some $s \in T \backslash\left\{t_{1}, t_{2}, \cdots, t_{n-1}\right\}$. If $g\left(t_{1}\right)=0$, set $g_{1}=g$ and we are done. If $g\left(t_{1}\right) \neq 0$, then $g\left(t_{1}\right)<0$.

Proceeding exactly as in the proof of Proposition 2.7 we obtain a function $g_{0} \in G \backslash\{0\}$ satisfying $g_{0}(t) g(t) \geqq 0$ for all $t \in T \backslash\left\{t_{1}\right\}$ and $g_{0}\left(t_{1}\right) \geqq$ 0 . If $g_{0}\left(t_{1}\right)=0$, set $g_{1}=g_{0}$. If $g_{0}\left(t_{1}\right) \neq 0$, then $g_{0}\left(t_{1}\right)>0$ and set $g_{1}=g-\alpha g_{0}$, where $\alpha=\left(g\left(t_{1}\right) / g_{0}\left(t_{1}\right)\right)<0$. Then $(-1)^{i} g_{1}(t) \geqq 0$ for all $t \in\left[t_{i}, t_{i+1}\right) \cap T(i=0,1, \cdots, n-1)$, and $g_{1}\left(t_{i}\right)=0$. If $g_{1}=0$, then $g=\alpha g_{0}$. Hence

$$
0 \leqq g_{0}(s) g(s)=\frac{1}{\alpha}[g(s)]^{2}<0
$$


which is absurd. Thus $g_{1} \neq 0$ and we are done.

Example 2.4 above, along with Theorems 3.1 and 4.1, show that properties (A-1) and (A-2) are not the same in general. However, there is one important case when they are the same.

Corollary 4.2. Let $I$ be any interval in $R$ and $G$ an $n$ dimensional subspace in $C_{0}(I)$. Then the following statements are equivalent:

(1) $G$ has any one of the equivalent properties (W-1), (W-1'), (W-2), (W-2'), (W-3), or (W-4);

(2) For each $f \in C_{0}(I)$, there exists $g_{0} \in P_{G}(f)$ such that $f-g_{0}$ has at least $n+1$ alternating peak points;

(3) For each $f \in C_{0}(I)$ which has a unique best approximation $g_{0} \in G, f-g_{0}$ has at least $n+1$ alternation peak points;

(4) Each $f \in C_{0}(I)$ with $P_{G}(f)=\{0\}$ has at least $n+1$ alternating peak points.

Proof. Corollary 2.9, Theorem 3.1, and Theorem 4.1.

In the particular case when $I$ is the compact interval $[a, b]$, the equivalence of properties (W-2'), (W-3), (W-4), (A-1), and (A-2) was first proved by Jones-Karlovitz [6].

\section{A characterization of property (A-3).}

THEOREM 5.1. Let $G$ be an $n$ dimensional subspace of $C_{0}(T)$. The following statements are equivalent.

(1) $G$ is Chebyshev and has one of the equivalent properties $(\mathrm{W}-2),\left(\mathrm{W}-2^{\prime}\right),(\mathrm{W}-3)$, or $(\mathrm{W}-4)$;

(2) For each $f \in C_{0}(T)$ and each $g_{0} \in P_{G}(f), f-g_{0}$ has at least $n+1$ alternating peak points.

Proof. $\quad(1) \Rightarrow(2)$. This follows from Theorem 4.1.

$(2) \Rightarrow(1)$. If statement (2) holds, Theorem 4.1 implies that $G$ has each of the equivalent properties (W-2), (W-2'), (W-3), and (W-4). If $G$ were not Chebyshev, $G$ would not be Haar so there would exist some nonzero $g_{0} \in G=\operatorname{span}\left\{g_{1}, g_{2}, \cdots, g_{n}\right\}$ which vanishes on a set of $n$ distinct points $T_{0}=\left\{t_{1}, t_{2}, \cdots, t_{n}\right\}$ of $T$. We may assume $\left\|g_{0}\right\|=1$. Then $\operatorname{det}\left[g_{i}\left(t_{j}\right)\right]_{1}^{n}=0$ so there are scalars $\alpha_{i}$ not all zero such that $\sum_{i=1}^{n} \alpha_{i} g_{j}\left(t_{i}\right)=0(j=1,2, \cdots, n)$, i.e., $\sum_{1}^{n} \alpha_{i} g\left(t_{i}\right)=0$ for all $g \in G$. Let $\sigma_{i}=\operatorname{sgn} \alpha_{i}$ if $\alpha_{i} \neq 0$ and $\sigma_{i}=1$ if $\alpha_{i}=0$. Since each $t_{i}$ is a $G_{j}$, Urysohn's lemma (or our linearization procedure) implies that for each $i=1,2, \cdots, n$, there exist $f_{i} \in C_{0}(T)$ and disjoint neighborhoods $U_{i}$ of $t_{i}$ such that $0 \leqq f_{i} \leqq 1, f_{i}\left(t_{i}\right)=1, f_{i}=0$ off $U_{i}$, and $f_{i}(t)<1$ 
for all $t \neq t_{i}$ (see e.g., [3; Cor. 4.2, p. 148]). Let $z=\sum_{1}^{n} \sigma_{i} f_{i}$. Then $z \in C_{0}(T), z\left(t_{i}\right)=\sigma_{i}(i=1,2, \cdots, n)$, and $|z(t)|<1$ if $t \in T \backslash T_{0}$. Set $f=z\left(1-\left|g_{0}\right|\right)+g_{0}$. Then $f \in C_{0}(T), f\left(t_{i}\right)-g_{0}\left(t_{i}\right)=z\left(t_{i}\right)=\sigma_{i}(i=1,2$, $\cdots, n)$, and

$$
\begin{aligned}
\left|f(t)-g_{0}(t)\right| & =|z(t)|\left(1-\left|g_{0}(t)\right|\right) \\
& =\left\{\begin{array}{cll}
<1 & \text { if } t \in T \backslash T_{0} \\
1 & \text { if } & t \in T_{0} .
\end{array}\right.
\end{aligned}
$$

Thus $\left\|f-g_{0}\right\|=1$ and the set of peak points for $f-g_{0}$ is $T_{0}$. If $g \in G$ and $\|f-g\|<1$, then since $\left|f\left(t_{i}\right)\right|=1$ for all $i$,

$$
\operatorname{sgn} g\left(t_{i}\right)=\operatorname{sgn} f\left(t_{i}\right)=z\left(t_{i}\right)=\sigma_{i} \quad(i=1,2, \cdots, n)
$$

so

$$
0=\sum_{1}^{n} \alpha_{i} g\left(t_{i}\right)=\sum_{1}^{n}\left|\alpha_{i}\right|\left|g\left(t_{i}\right)\right|>0
$$

which is absurd. Thus $\|f-g\| \geqq 1=\left\|f-g_{0}\right\|$ for all $g \in G$ and hence $g_{0} \in P_{G}(f)$. But $f-g_{0}$ has only $n$ peak points (viz. the set $T_{0}$ ). This contradiction to (2) shows that $G$ must be Chebyshev.

In a result related to Theorem 5.1, Gopinath and Kurshan [4] essentially proved that an $n$ dimensional subspace $G$ of $C_{0}(T)$ is Chebyshev and has property $(\mathrm{W}-4) \Leftrightarrow$ it has the property $(G-K)$ : For each set of points $-\infty=t_{0}<t_{1}<\cdots<t_{n-1}<t_{n}=\infty$ with $t_{i} \in$ $T(i=1,2, \cdots, n-1)$, there exists $g \in G$ such that

$$
(-1)^{i} g(t)>0 \text { if } t \in\left(t_{i}, t_{i+1}\right) \cap T
$$

$(i=0,1, \cdots, n-1)$, and

$$
g\left(t_{i}\right)=0 \quad(i=1,2, \cdots, n-1) .
$$

Theorem 5.1 can be strengthened in case $T$ is an interval.

Corollary 5.2. Let $I$ be an interval in $R$ and $G$ an $n$ dimensional subspace of $C_{0}(I)$. Then the following statements are equivalent:

(1) $G$ is Chebyshev;

(2) For each $f \in C_{0}(I)$ and each $g_{0} \in P_{G}(f), f-g_{0}$ has at least $n+1$ alternating peak points;

(3) For each $f \in C_{0}(I)$ and each $g_{0} \in P_{G}(f), f-g_{0}$ has at least $n+1$ peak points.

Proof. $\quad(1) \Rightarrow(2)$. By Corollary $3.2(\mathrm{c}), G$ has all the weak Chebyshev properties so the result follows from Theorem 5.1.

$(2) \Rightarrow(3)$ is obvious. 
$(3) \Rightarrow(1)$. This follows exactly as in the proof of $(2) \Rightarrow(1)$ in Theorem 5.1.

REMARK 5.3. The implication $(1) \Rightarrow(2)$ in Corollary 5.2 , in the particular case when $I=[a, b]$, is just the classical alternation theorem. Also, when $I=[a, b]$, the implication $(2) \Rightarrow(1)$ was proved by Handscomb, Mayers, and Powell [5]. We now show that implication (1) $\Rightarrow$ (2) fails in general if $I$ is not an interval. Indeed, it fails if one adjoins a single point outside the interval.

EXAMPLE 5.4. Let $T=[a, b] \cup\{c\}$, where $c \notin[a, b]$. Let $G=$ span $\left\{g_{1}\right\}$, where $g_{1}(t)=1$ if $t \in[a, b]$ and $g_{1}(c)=-1$. Then $G$ is Chebyshev in $C(T)$ since $g_{1}$ has no zeros. But if $f(t) \equiv 1$, then $P_{G}(f)=0$ and $f-P_{G}(f)$ does not have two alternating peak points.

6. Examples of weak Chebyshev subspaces. There are a number of important subspaces which are not Chebyshev but are weak Chebyshev. We give a few examples below.

6.1. (Polynomial Splines). Let $T=[a, b]$ and fix any $k(\geqq 1)$ points $s_{1}<s_{2}<\cdots<s_{k}$ in $T$. For any integer $m \geqq 0$ let

$$
S_{m, k}=\operatorname{span}\left\{1, t, \cdots, t^{m},\left(t-s_{1}\right)_{+}^{m}, \cdots,\left(t-s_{k}\right)_{+}^{m}\right\},
$$

where

$$
(t-s)_{+}^{m}=\left\{\begin{array}{cll}
(t-s)^{m} & \text { if } \quad t \geqq s \\
0 & \text { if } \quad t<s .
\end{array}\right.
$$

$S_{m, k}$ is the $n \equiv m+k+1$ dimensional subspace of $C[a, b]$ known as "the polynomial splines of degree $m$ with $k$ fixed knots." It is known (see e.g., [7], p. 18) that $S_{m, k}$ has property (W-3), and thus by Corollary 4.2 has all of the weak Chebyshev properties (W-i) $\left(i=1,1^{\prime}, 2,2^{\prime}, 3\right.$, and 4) as well as the alternation properties (A-1) and (A-2).

6.2. (Weighted Polynomial Splines). The example in (1) can be modified as follows. Let $T=R$ and $k$ points $s_{1}<s_{2}<\cdots<s_{k}$ in $T$ be given. Let $w \in C_{0}(T)$ be any positive function such that $w \cdot p \in$ $C_{0}(T)$ for any polynomial $p$ (e.g., $w(t)=e^{-t^{2}}$ ). Then the $n \equiv m+k+1$ dimensional subspace

$$
S_{m, k}^{0}=\left\{w g \mid g \in S_{m, k}\right\},
$$

where $S_{m, k}$ is defined as in 6.1 , obviously has property (W-3) since $S_{m, k}$ does. Thus by Corollary $4.2, S_{m, k}^{0}$ has all the Chebyshev properties $(\mathrm{W}-\mathrm{i})\left(\mathrm{i}=1,1^{\prime}, 2,2^{\prime}, 3\right.$, and 4$)$ and the alternation properties (A-1) and $(\mathrm{A}-2)$. 
6.3. (Weighted Chebyshev subspace). Let $T=I$ be any interval in $\boldsymbol{R}$ and $P$ any $n$ dimensional Chebyshev subspace of $C_{0}(T)$. Let $w \in C_{0}(T)$ be any nonnegative function which does not vanish identically, and set

$$
G=\{w p \mid p \in P\} .
$$

Then since $P$ is Chebyshev, it follows that $G$ is an $n$ dimensional subspace of $C_{0}(T)$ having property (W-3), and hence, by Corollary 4.2 , all the weak Chebyshev properties (W-i) $\left(i=1,1^{\prime}, 2,2^{\prime}, 3\right.$, and 4) as well as the alternation properties (A-1) and (A-2).

6.4. Let $T=N$ denote the set of natural numbers (so $C_{0}(T)=c_{0}$ ) and let any $n$ points $k_{1}<k_{2}<\cdots<k_{n}$ in $T$ be given. Define $g_{i} \in c_{0}$ by

$$
g_{i}(t)= \begin{cases}1 & \text { if } t=k_{i} \\ 0 & \text { otherwise }\end{cases}
$$

$(i=1,2, \cdots, n)$. Then $G=\operatorname{span}\left\{g_{1}, g_{2}, \cdots, g_{n}\right\}$ is an $n$ dimensional subspace of $c_{0}$ which is easily seen to have properties (W-1) and (W-2). Thus by Proposition 2.5, Lemma 2.2, and Theorems 3.1 and $4.1, G$ has all the weak Chebyshev properties (W-i) $\left(i=1,1^{\prime}, 2,2^{\prime}, 3\right.$, and 4) and the alternation properties (A-1) and (A-2).

We note that none of the above four examples is a Chebyshev subspace in general.

7. A generalization. We can give the following generalization of the equivalence $(1) \Leftrightarrow(3)$ of Corollary 5.2. In particular, it provides another characterization of Chebyshev subspaces in $C_{0}(T)$ for certain $T$ (including $T$ metric). However, unlike Haar's characterization concerning the number of zeros of elements of the subspace, our characterization is not intrinsic.

THEOREM 7.1. Let $T$ be any locally compact Hausdorff space containing at least $n+1$ points and let $G$ be an $n$ dimensional subspace of $C_{0}(T)$. If each point of $T$ is a $G_{o}$ (e.g., if $T$ is metric), or if each nonzero element of $G$ has only finitely many zeros, then the following statements are equivalent:

(1) $G$ is Chebyshev;

(2) For each $f \in C_{0}(T)$ and each $g_{0} \in P_{G}(f), f-g_{0}$ has at least $n+1$ peak points.

The proof of the implication $(2) \Rightarrow(1)$ is similar to the proof of the implication $(2) \Rightarrow(1)$ of Theorem 5.1. The implication $(1) \Rightarrow(2)$ 
is well-known and due, for compact $T$, to Remez (see e.g., [8]).

It is worth noticing that this characterization of Chebyshev spaces is no longer valid in general if both the conditions on $T$ and $G$ (viz. (i) each point of $T$ be a $G_{\delta}$, and (ii) each nonzero element of $G$ have only finitely many zeros) are dropped. To see this, let $\beta \boldsymbol{R}$ denote the Stone-Čech compactification of $\boldsymbol{R}$ and consider the space $T=$ $\beta \boldsymbol{R} \backslash \boldsymbol{R}$. It is well-known that $T$ is a compact Hausdorff space in which no point is a $G_{\delta}$ (see e.g., [9; p. 150, prob 112]). A simple induction shows that no finite subset of $T$ is a $G_{\dot{o}}$. Thus each $f \in$ $C(T)$ has an infinity of peak points (since the set of all peak points is a $G_{\delta}$ ). By Urysohn's lemma there exists a nonzero $g \in C(T)$ which has a zero. Thus $G=\operatorname{span}\{g\}$ is not Chebyshev, but for each $f \in$ $C(T)$ and $g_{0} \in P_{G}(f), f-g_{0}$ has infinitely many peak points.

\section{REFERENCES}

1. N. Bourbaki, Éléments de Mathématique, Livre III, Topologie Ǵenérale, Chs. 1-2, Hermann, Paris, 1965.

2. J. Bram, Chebyshev approximation in locally compact spaces. Proc. Amer. Math. Soc., 9 (1958), 133-136.

3. J. Dugundji, Topology, Allyn and Bacon, Boston, 1966.

4. B. Gopinath and R. P. Kurshan, The existence in $T$-spaces of functions with prescribed alternations, J. Approximation Theory, 21 (1977), 143-150.

5. D. C. Handscomb, D. F. Mayers, and M. J. D. Powell, The general theory of linear approximation, in Methods of Numerical Approximation (ed. by D. C. Handscomb), Pergamon Press, New York, 1966.

6. R. C. Jones and L. A. Karlovitz, Equioscillation under nonuniqueness in the approximation of continuous functions, J. Approximation Theory, 3 (1970), 138-145.

7. S. Karlin and W. J. Studden, Tchebycheff Systems: With Applications in Analysis and Statistics, Interscience, New York, 1966.

8. I. Singer, Best Approximation in Normed Linear Spaces by Elements of Linear Subspaces, Springer, New York, 1970.

9. A. Wilansky, Topology for Analysis, Ginn, Waltham, Mass., 1970.

10. R. Zielke, Einige Eigenschaften schwacher Tschebyscheff-Systeme, preprint (1977).

Received August 2, 1978 and in revised form July 12, 1979. The first author was supported in part by NSF Grant No. MCS 77-07582. The work of the second (resp. third) named author was performed while he was on leave from the Institut fur Angewandte Mathematik, der Universität Erlangen-Nürnberg, Germany (resp. National Institute for Scientific and Technical Creation, Bucharest, Romania) and in residence Sept. 1976-Sept. 1977 (resp. May-June 1977) at The Pennsylvania State University. The second author gratefully acknowledges useful conversations with Professor Hubert Berens.

Department of Mathematics

The Pennsylvania State University

UNIVERSITY PARK, PA 16802 



\section{PACIFIC JOURNAL OF MATHEMATICS}

\section{EDITORS}

DONALD BABBITT (Managing Editor)

University of Galifornia

Los Angeles, California 90024

Hugo RossI

University of Utah

Salt Lake City, UT 84112

C. C. MoOre AND ANDrew OGG

University of California

Berkeley, CA 94720

\section{J. DugundjI}

Department of Mathematics University of Southern California

Los Angeles, California 90007

R. Finn and J. Milgram

Stanford University

Stanford, California 94305

ASSOCIATE EDITORS

E. F. Beckenbachi

B. H. NeumanN

F. WOLF

K. YOSHIDA

\section{SUPPORTING INSTITUTIONS}

UNIVERSITY OF BRITISH COLUMBIA

CALIFORNIA INSTITUTE OF TECHNOLOGY

UNIVERSITY OF CALIFORNIA

MONTANA STATE UNIVERSITY

UNIVERSITY OF NEVADA, RENO

NEW MEXICO STATE UNIVERSITY

OREGON STATE UNIVERSITY

UNIVERSITY OF OREGON
UNIVERSITY OF SOUTHERN CALIFONIA

STANFORD UNIVERSITY

UNIVERSITY OF HAWAII

UNIVERSITY OF TOKYO

UNIVERSITY OF UTAH

WASHINGTON STATE UNIVERSITY

UNIVERSITY OF WASHINGTON

Printed in Japan by International Academic Printing Co., Ltd., Tokyo, Japan 


\section{Pacific Journal of Mathematics \\ Vol. 89 , No. 1 \\ May, 1980}

David Bressoud, A note on gap-frequency partitions ................ 1

John David Brillhart, A double inversion formula ................. 7

Frank Richard Deutsch, Günther Nürnberger and Ivan Singer, Weak

Chebyshev subspaces and alternation .......................

Edward Richard Fadell, The relationship between Ljusternik-Schnirelman

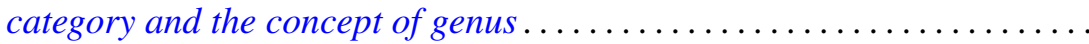

Harriet Jane Fell, On the zeros of convex combinations of polynomials.......

John Albert Fridy, An addendum to: "Tauberian theorems via block

dominated matrices" ..................................

Andrzej Granas, Ronald Bernard Guenther and John Walter Lee, Applications of topological transversality to differential equations. I. Some nonlinear

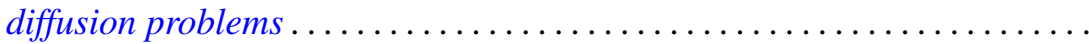

David E. Handelman and G. Renault, Actions of finite groups on self-injective

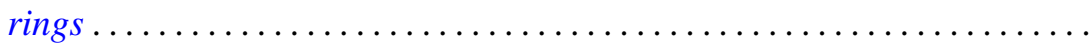

Michael Frank Hutchinson, Local $\Lambda$ sets for profinite groups .............

Arnold Samuel Kas, On the handlebody decomposition associated to a

Lefschetz fibration...

Hans Keller, On the lattice of all closed subspaces of a Hermitian space.....

P. S. Kenderov, Dense strong continuity of pointwise continuous

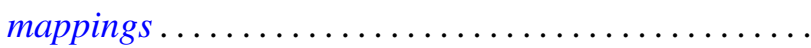

Robert Edward Kennedy, Krull rings.................

Jean Ann Larson, Richard Joseph Laver and George Frank McNulty,

Square-free and cube-free colorings of the ordinals ...

Viktor Losert and Harald Rindler, Cyclic vectors for $L^{p}(G)$

John Rowlay Martin and Edward D. Tymchatyn, Fixed point sets of

1-dimensional Peano continua...

Augusto Nobile, On equisingular families of isolated singularities ...

Kenneth Joseph Prevot, Imbedding smooth involutions in trivial bundles ...

Thomas Munro Price, Spanning surfaces for projective planes in four space.

Dave Riffelmacher, Sweedler's two-cocycles and Hochschild cohomology....

Niels Schwartz, Archimedean lattice-ordered fields that are algebraic over

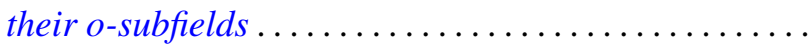

Chao-Liang Shen, A note on the automorphism groups of simple dimension groups.

Kenneth Barry Stolarsky, Mapping properties, growth, and uniqueness of

Vieta (infinite cosine) products ...

Warren James Wong, Maps on simple algebras preserving zero products. I.

The associative case ............................. 